\title{
Retrospective cohort mortality study of workers at an aircraft maintenance facility. II Exposures and their
} assessment

\author{
P A Stewart, J S Lee, D E Marano, R Spirtas, C D Forbes, A Blair
}

\begin{abstract}
Methods are presented that were used for assessing exposures in a cohort mortality study of 15000 employees who held 150000 jobs at an Air Force base from 1939 to 1982 . Standardisation of the word order and spelling of the job titles identified 43000 unique job title organisation combinations. Walkthrough surveys were conducted, long term employees were interviewed, and available industrial hygiene data were collected to evaluate historic exposures. Because of difficulties linking air monitoring data and use of specific chemicals to the departments identified in the work histories, position descriptions were used to identify the tasks in each job. From knowledge of the tasks and the chemicals used in those tasks the presence or absence of 23 chemicals or groups of chemicals were designated for each job organisation combination. Also, estimates of levels of exposure were made for trichloroethylene and for mixed solvents, a category comprising several solvents including trichloroethylene, Stoddard solvent, carbon tetrachloride, $\mathbf{J P}_{4}$ gasoline, freon, alcohols, 1,1,1-trichloroethane, acetone, toluene, methyl ethyl ketone, methylene chloride, o-dichlorobenzene, perchloroethylene, chloroform, styrene, and xylene.
\end{abstract}

US National Cancer Institute, NIH

P A Stewart, R Spirtas, A Blair

University of Utah

J S Lee, D E Marano

Currently with Industrial Health Inc

D E Marano

Currently with the National Institute of Child Health and Human Development, NIH

R Spirtas

Westat Inc

C D Forbes

Currently with the General Accounting Office, US Congress

C D Forbes
Developing meaningful exposure categories is one of the most difficult problems in conducting studies on occupational epidemiology. It is a necessary step, however, in establishing an exposure response relation, an important criterion for assessing causality. Usually three steps are necessary in assessing exposure for occupational studies: creation of a job dictionary, evaluation of historical exposures, and development and application of a method of assessment of exposure. The first two are fairly straightforward and are generally performed similarly by most investigators. The third step, however, depends upon the quality of the available work histories and exposure information, and the time and cost constraints of the study. Previous studies ${ }^{1-12}$ have reported several approaches including ever never evaluations based on the type of plant or department, semiquantitative estimates (high, medium, low) by department or by job, or quantitative estimates by job using air monitoring results.

This paper describes the methods used to assess historical exposures for a retrospective cohort study, evaluating the mortality experience of 15000 workers at an aircraft maintenance facility. ${ }^{13}$ Air monitoring results were available back to the 1960 s, but they often could not be linked to specific jobs because of the lack of information on department in the work histories. Other sources of information had to be relied upon to allow assignment of exposures to individual job, department, and year combinations. As a result, the records were sufficient for quantification of only two types of exposures, trichloroethylene and mixed solvents, and for identification of only the presence or absence of other chemicals.

The paper also briefly describes some of the operations being performed at this facility and the associated exposures. Since the inception of the facility in 1939 workers have performed a variety of tasks that involved several potentially hazardous chemical exposures, including paint stripping, spray painting, soldering, and cleaning of mechanical and electronic parts with solvents. The exposure of primary interest in this investigation was to organic solvents, particularly trichloroethylene (TCE). 
Table 1 Example of a work history

\begin{tabular}{|c|c|c|c|c|c|c|}
\hline Job title & Directorate & Division & Branch & Section & Unit & Year started \\
\hline $\begin{array}{l}\text { Junior aircraft sheet metal worker } \\
\text { Senior aircraft metal surface repairer } \\
\text { AC Sheet metal manufacture and repair }\end{array}$ & $\begin{array}{l}\text { Engineering } \\
\text { Maintenance }\end{array}$ & & & $\begin{array}{l}\text { Manufacture } \\
\text { and repair }\end{array}$ & Repair & $\begin{array}{l}1942 \\
1944 \\
1950\end{array}$ \\
\hline $\begin{array}{l}\text { Training officer } \\
\text { AC sheet metal inspector } \\
\text { AC sheet metal inspector } \\
\text { AC sheet metal work inspector }\end{array}$ & & & & & & $\begin{array}{l}1951 \\
1953 \\
1955 \\
1956\end{array}$ \\
\hline
\end{tabular}

\section{Methods}

The cohort included all civilian employees who worked at an air force base for at least one year between 1952 and $1956 .{ }^{13}$ The base, which serves as a major supply and maintenance depot, is organised into "directorates," each of which is subdivided into several other organisational levels, the lowest of which will be designated as a unit. It is only at the unit level where exposures can be identified, because two units under the same organisation can be performing different operations, and therefore have different exposures. Job titles may be similar or different. Organisation names at all levels have changed many times throughout the history of the base and a complete record of these changes was not maintained. As well as the formal organisational hierarchy, a level of organisation exists called "shop," a work area with a designated function which may or may not correspond to the unit. Shops are important because industrial hygiene data were collected and retained by shop and the documentation often did not identify the corresponding organisation.

\section{CREATION OF A JOB DICTIONARY}

The job dictionary was created by abstracting from each employee's official personnel folder (OPF) information on job titles, the names of the organisations at the different levels and dates. Extensive and comprehensive information on job titles and corresponding dates was available; however, information on organisation title varied by OPF, job, and calendar year and was usually incomplete. Moreover, shop was not identified. Table 1 shows an example of a typical work history.

As is the usual practice with personnel records, when an organisation changed names, no modification was made to the work history records of individual workers although a worker who entered the organisation after the change of name would have a personnel record that would reflect the change. Consequently, with the many changes in organisation names and often the lack of information on unit, it was difficult to identify the unit in which a job was assigned at any given moment in time.

To develop a record of the changes in the organisa- tions and shops, other historical documents retained by the base were collected and reviewed. These included directories of organisations, telephone books for the facility, organisational charts, and textual histories of the base. These documents usually contained some information on the higher levels of organisations but little information on units or shops. None, however, provided a complete picture of all operations performed at the base. As a result, histories of the units and their corresponding shop titles remained incomplete.

The impact of these peculiarities can be seen in a typical example. Table 2 shows the history of one of the most documented shops, the plastic shop, as well as could be determined. No information on unit was available before 1953. For a person who started working in the plastic shop in 1951, his OPF may have identified him as having worked in the higher level sheet metal branch rather than a lower organisational level, such as the unit title for the plastic shop. If this employee did not change jobs the 1951 entry would remain as his most recent job, even if he was still working there in 1971, by which time the organisation name had changed several different times. By contrast, the history of another employee who started working in the same shop in 1971 may have indicated the plastic and glass unit. Thus, although these two may have worked next to each other in 1971, their histories could indicate that they worked in what appeared to be different organisations.

Table 2 Summary of information collected on changes in the name of the plastic shop

\begin{tabular}{ll}
\hline $\begin{array}{l}\text { Year of } \\
\text { change }\end{array}$ & Organisation name \\
\hline 1983 & Plastic manufacturing and repair (level unknown) \\
1977 & Plastic unit \\
1972 & Plastic and glass unit \\
1971 & Plastic and glass unit \\
1970 & Plastic and glass unit \\
1969 & Plastic and glass unit \\
1955 & Plastic and fibreglass unit \\
1953 & Plastic unit \\
1952 & Sheet metal fuselage section \\
1949 & Sheet metal branch \\
1943 & Plastic and plexiglas (level unknown) \\
\hline
\end{tabular}


A job dictionary of approximately 150000 combinations of job titles and their most specific (lowest level) organisation was created from the work histories abstracted from the OPFs. Removal of duplicate titles and standardisation of spelling and word order reduced the number of unique job organisation combinations to 43000 . Further grouping of job titles by unit could not be accomplished because the same job title was found in several different units. Thus the final job dictionary consisted of 43000 job titles associated with varying levels of organisations.

\section{DETERMINATION OF THE OPERATIONS AND EXPOSURES}

Several sources of information were used to identify what exposures had occurred on the base. Industrial hygiene results had been retained by the air force since the mid-1960s. Included in these files were air, noise, radiation, and ventilation measurements. The air monitoring measured both personal and area concentrations and, in the early years, samples were of short duration. Sampling methods were generally not specified, although occasionally the use of detector tubes was noted.

Monitoring was generally conducted either as a result of complaints or as part of annual industrial hygiene surveys. In recent years more full shift samples were taken and documentation was more complete. Reports on walkthrough surveys and chemical inventories were also available. These reports usually provided general information on the number of persons in the shop, reports of illnesses, and job titles.

Worker compensation files were also used in the evaluation of exposures. Thesefiles identified job title, organisation, job duties, historical working conditions, and in some cases, the exposure histories of employees who filed for worker compensation. About 100 of these files existed dating back to the 1950s. Since the mid-1960s technical orders were available that identified procedures to be followed during the repair of specific parts. Often these specified the chemicals to be used.

Each employee in the federal government has a written description of job duties and tasks to be performed in that job. These are maintained in the person's OPF throughout his government career. A complete file of historical position descriptions had not been maintained, but roughly 8000 of these were collected during the study. The PDs identified the job, organisation, date, tasks, and sometimes specific chemicals.

As well as reviewing the available air force records, two experienced industrial hygienists (JSL, DEM) conducted walkthrough surveys of the shops and interviewed long term employees about changes in the operations, chemicals used, number of employees, job titles and tasks, and control measures.
DESCRIPTION OF THE OPERATIONS AND EXPOSURES

In the early years of the base (1939-1954), solvents, primarily Stoddard solvent, carbon tetrachloride, trichloroethylene, and alcohols, were used liberally to clean metal parts. Of these, Stoddard solvent was used most frequently and could be found throughout the base at wash stations or in open buckets. Towards the end of this period, buckets were replaced with fire safety cans.

Carbon tetrachloride and various alcohols were also used in smaller amounts for cleaning small parts. Alcohols were used more in the electronics shops, whereas carbon tetrachloride was used more in the instrument shops. Both solvents were available in open cans at each work bench, and the worker applied the solvent with either a cloth or a brush as often as 15 times a day. The duration of exposure for each application was about five or 10 minutes, and exposure concentrations probably rarely exceeded 10 to 15 parts per million (ppm), an estimate based on the more recent air monitoring results and the lack of any major changes in engineering controls or work practices over time. Soldering also was performed in these operations as was minor spray painting. In many other shops throughout the base employees also soldered and used small amounts of different solvents for cleaning purposes during the repair of electrical or mechanical instruments, including training devices, missile electronics, navigational instruments, and precision measurement equipment.

Two shops are of particular interest in this period because of the results of the mortality analysis. ${ }^{13}$ In the parachute shop, cotton parachutes were dry cleaned using carbon tetrachloride. Stoddard solvent and toluene may also have been used to clean the parachutes. In the late 1950 s perchloroethylene replaced carbon tetrachloride. In the second shop, the fabric and dope shop, fabric surfaces of planes were treated with nitrate cellulose dope, a stiffening agent. Also used in this shop were acetone, toluene, lacquer thinner, and nitrate cellulose paint. The components of the dope, lacquer thinner, and paint are not known. Nitrocellulose resins can contain solvents, including alcohols, acetates, toluene, naphtha, and benzene. ${ }^{14} 15$

Aircraft repair lines were always located in the main hangars, and, although the types of aircraft changed through time, the maintenance activities and solvent exposure patterns remained reasonably constant. The main repair activities in this area were performed by mechanics, electricians, and sheet metal workers. In the early years Stoddard solvent was probably the principal chemical used. Trichloroethylene was introduced around 1955 and in 1968 , to reduce the potential of fire, 1,1,1-trichloroethane replaced the previously used solvents in this operation.

Aircraft mechanics used large amounts of solvents 
when spraying or wiping hydraulic lines with rags to remove grease and oils before checking for leaks. Short but high exposures occurred when mechanics worked in the semiconfined space of the wing sections. Sheet metal workers had a slight exposure to solvents while spot cleaning aircraft surfaces and during the 1950s, to zinc chromate paint for spot painting. Electricians used methylene chloride when depotting electrical connections in the cockpits during the $1970 \mathrm{~s}$. This confined space activity resulted in high exposure levels. The workers on the repair lines and other aircraft workers had a potential exposure to high octane gasoline and, later, to $\mathrm{JP}_{4}$ jet fuel, particularly before the late $1950 \mathrm{~s}$, when the fuel systems of the aircraft were not purged before entering the hangars.

Trichloroethylene was the predominant solvent used in the few vapour degreasers present in the shops during the early years. These degreasers were located in the electroplating shop in the main hangar, the propeller shop, and the engine repair shop; also, there may have been a small amount of degreasing in the instrument shops.

In the mid-1950s several major changes took place in patterns of solvent use. Due to the potential fire hazard associated with Stoddard solvent, the use of TCE as a cold state solvent increased. As TCE became more available, carbon tetrachloride was needed less, and it stopped being used around the same time. Employment of TCE differed little from the pattern described earlier for carbon tetrachloride and alcohols.

During the 1950s and 1960s the number of people exposed to TCE increased because of the growing use of TCE as a cold state solvent and as a degreaser. During the early part of this period, degreasers were not well controlled. ${ }^{16}$ For example, the lids were left open when the degreaser was not in use; some degreasers had malfunctioning or unused cooling jackets, and none had water separators. Industrial hygiene sampling data from the early 1960 s showed that potential levels of worker exposure to TCE were as high as $400 \mathrm{ppm}$ during the 10 to 15 minutes that the worker was degreasing the parts. The frequency of degreaser use by workers varied from shop to shop. In the engine shop or the propeller shop, workers used degreasers a few times a week, whereas in the electroplating shop, the wheels, brakes, and strut shops, and the hydraulics shop, degreasers were used four to five times a day. In the mid-1960s, an industrial hygiene evaluation of all vapour degreasers was conducted and efforts were made to reduce these exposure concentrations. ${ }^{17} \mathrm{By}$ the end of the $1960 \mathrm{~s}$ exposure to trichloroethylene had been reduced significantly. ${ }^{17}$ Industrial hygiene sampling data during this period show that workers using the vapour degreasers were exposed to TCE concentrations averaging around $200 \mathrm{ppm}$ during the 15 minutes they normally took to clean parts. In 1979 TCE was replaced by 1,1,1-trichloroethane as the primary solvent used in vapour degreasers throughout the base.

Also during the 1960s in the bench work operations, other chemicals including toluene, xylene, acetone, and methyl ethyl ketone were employed more often. Initially, these solvents were kept on the work bench in glass bottles, but later they were contained in glass bottles with a pump and a reservoir on the top for the solvent. In the early 1970 s, squeeze bottles were adopted. Although any of these solvents could be found at a given work station, the specific solvent found at any time and the amount used varied considerably. Generally, however, the alcohols and non-chlorinated solvents were used four or five times a day in the electronics area and the chlorinated solvents about 15 times a day in the instrument shops.

In 1968 the base made a change by replacing TCE used as a cold state solvent with 1,1,1-trichloroethane. This switch was made base wide, with the exception of the fabric and parachute shop, where both perchloroethylene and trichloroethylene continued to be used interchangeably to spot clean fabric. Many other operations on the base resulted in exposures other than those already mentioned, but because they concerned fewer workers they have not been described.

\section{EXPOSURE ASSESSMENT}

The original intent of the study was to estimate historical levels of exposure to the chemicals found on the base for each job unit, and year combination. This approach, however, proved not to be feasible because (1) the monitoring results were collected by shop; (2) few monitoring results were available for any particular shop despite numerous air monitoring results dating back to the 1960 s; (3) the specific unit which corresponded to shop was often not known; and (4) the work history records did not usually identify the unit. Because of these limitations it was not possible to estimate levels of exposure and it was often not possible to identify specific solvents used.

Because the work history records were insufficient to identify jobs to organisations with exposures, position descriptions (PDs) became the primary source of data on which exposure assessments were based. Thus potential exposures for some jobs were identified in the PD; for others they were deduced from the task. For example, by knowing that the airframe disassembler washers stripped paint off planes, exposure to methylene chloride was identified. For many jobs, however, specific exposures could not be identified, because the same job title occurred in different shops where different chemicals were used. A further complication was that solvents used for 
Table 3 Frequency and duration of use of TCE and mixed solvents

\begin{tabular}{lll}
\hline Types of job & Frequency $(f)$ & $\begin{array}{c}\text { Duration } \\
(d ; \text { min) }\end{array}$ \\
\hline $\begin{array}{l}\text { Benchwork } \\
\text { (low level exposures) }\end{array}$ & $\begin{array}{l}\text { Continuous (about 15/day) } \\
\text { Intermittent (about four/ } \\
\text { day) }\end{array}$ & 5 \\
$\begin{array}{c}\text { Vapour degreasing } \\
\text { (peak exposures) }\end{array}$ & $\begin{array}{l}\text { Frequent (two/day) } \\
\text { Infrequent (two/ } \\
\text { week =0.4/day) }\end{array}$ & 15 \\
\hline
\end{tabular}

specific tasks had changed frequently, but a complete record was not available identifying these changes. Consequently, it was not possible in many instances to link specific solvents with specific jobs. A category of "mixed solvents" for these jobs, therefore, was developed which encompassed the potential use of one or more of the following: trichloroethylene, Stoddard solvent, carbon tetrachloride, $\mathrm{JP}_{4}$ gasoline, freon, alcohols, 1,1,1-trichloroethane, acetone, toluene, methyl ethyl ketone, methylene chloride, odichlorobenzene, perchloroethylene, chloroform, styrene, and xylene. Examples of jobs with potential for mixed solvent exposures included elecrical instrument repair, hardware repair, and sheet metal repair.

Estimating levels of exposure proved more difficult than identifying the specific chemicals used, primarily because it was not possible to link shop designated in the monitoring data to the unit designated in the work history records and because few monitoring data existed for each shop. For two exposures, TCE and mixed solvents, however, a more detailed approach was possible. As described above, both exposures resulted from the bench work operations of cleaning and repairing small parts. Relying primarily on the PDs, but also using the technical orders and the histories of the shop (table 2), the industrial hygienists were able to distinguish different patterns of exposures for these two types of benchwork exposures. The frequency ( $f$ ) of use was noted as continuous (used regularly throughout the day) or intermittent (used infrequently throughout the day) (table 3). Also, f of peak exposures to TCE using vapour degreasers could be identified from the PDs. Use of TCE at the degreasers was designated as frequent ( $\geq$ twice a day) or infrequent (about twice a week). Duration (d) of use in minutes was also estimated for these different operations for both mixed solvents and for TCE. In addition, for TCE, an index of exposure (i) was developed to reflect changes in exposures over time based on historical reports written by the base industrial hygienists (table 4). ${ }^{16}{ }^{17}$ The exposure levels for mixed solvents do not change over time, because no changes in engineering controls or work practises were identified.

To arrive at relative exposure levels by various jobs for TCE and mixed solvents, an equation was developed:

$$
I=\frac{(f)(d)(i)}{480}
$$

where: $f$ is the frequency of use (table 3); $\mathrm{d}$ is the duration of use (table 3 ); $i$ is the index of exposure (table 4); and 480 is the number of minutes in an eight hour workday.

To determine cumulative exposure for purposes of the mortality analysis the number of years spent at each job was multiplied by the index I. These products were added together to get cumulative exposure to TCE or mixed solvents for each person.

\section{Results}

Table 5 presents the number of people ever exposed to each chemical category. The categories are not mutually exclusive. Over two thirds of the cohort had potential exposure to solvents and about half had potential exposure to TCE. Stoddard solvent was the second most common single exposure and "other chemicals"-for example, acids-was the most commonly identified non-solvent category.

Among the 10256 workers exposed to mixed solvents, 8753 had intermittent exposure to mixed solvents and 6356 had continuous exposure. For those with only low level exposures to TCE, 4605 were in the intermittent category and 3292 were in the continuous category. For those with peak exposures to TCE, 1267 had infrequent exposures and 3322 had frequent exposures. Finally, 2358 had both low level and peak TCE exposure.

\section{Discussion}

Estimating historical exposure levels is difficult because often industrial hygiene measurements are missing or incomplete. As a result, various approaches to making exposure assessments have been published. Exposure groups have been created by using manufacturing process, ${ }^{1}$ or work area. ${ }^{2}$ Gamble and Spirtas $^{3}$ suggested grouping

Table 4 Indices used in exposure estimation

\begin{tabular}{llll}
\hline & \multicolumn{2}{l}{ Index } & \\
\cline { 2 - 3 } & $T C E^{\prime}$ & & \\
\cline { 2 - 3 } Period & Peak & Low level & Mixed solvents \\
\hline $1939-54$ & 600 & 10 & 10 \\
$1955-67$ & 400 & 10 & 10 \\
$1968-78$ & 200 & 0 & 10 \\
$1979-83$ & 0 & 0 & 10 \\
\hline
\end{tabular}

${ }^{1}$ Use of TCE was eliminated from the base as a cold solvent in 1968 and as a degreasing solvent in 1978 . 
Table 5 Chemicals at the base and number in cohort exposed

\begin{tabular}{lc}
\hline & No of people \\
Chemical & ever exposed \\
\hline Mixed solvents & 10256 \\
Trichloroethylene & 7282 \\
Stoddard solvent/kerosene & 6977 \\
Carbon tetrachloride & 6737 \\
Other chemicals & 4783 \\
JP & 3587 \\
Trichlorotrifluoroethane (freen) & 2469 \\
Solder flux & 2367 \\
Isopropyl alcohol & 2341 \\
Zinc chromate & 2327 \\
1,1,1-Trichloroethane & 2215 \\
Acetone & 2179 \\
Toluene & 1841 \\
Methyl ethyl ketone & 1769 \\
Methylene chloride & 1222 \\
Metal fumes/dust & 1110 \\
Ortho-dichlorobenzene & 953 \\
Perchlorethylene & 851 \\
Known or suspected carcinogens $\dagger$ & 832 \\
Other alcohols & 780 \\
Chloroform & 241 \\
Styrene & 169 \\
Nitroglycerine & 122 \\
Silica & 111 \\
Xylene & 104 \\
\hline
\end{tabular}

Total cohort 14457 .

^Acids, salts, etc.

†Asbestos, beryllium, cadmium, 4,4'methylene bis(2-chloroaniline).

occupational titles by machine, or process, or both and by the product being manufactured. These approaches were not taken because a more detailed assessment was desired than these methods allowed. Corn and Esmen ${ }^{4}$ further refined exposure classification by suggesting the use of exposure zones based on identification and similarity of tasks, exposure to a particular hazardous substance, and other aspects of the environment (other substances, ventilation, etc). In our study, however, exposure zones could not always be identified because of the inability to identify where jobs were located. Where many environmental measurements have existed (hundreds to thousands), investigators have estimated exposure levels, ${ }^{591112}$ but in our study the number of monitoring results was insufficient for this approach and it was not possible to link the results to many of the jobs in the study. Rice $e t a l^{11}$ who also had comparatively fewer data from each plant, calculated a mean exposure level for each job title across all the plants making the assumption that, although exposure levels varied for a specific job across plants, the variation would be less than that across jobs. We took a similar approach by assuming similar jobs across departments had the same potential exposures.

The approach used in this study is unusual among published reports quantifying exposure levels because the quality of information was barely adequate to estimate exposures. The initial goal was to make quantitative estimates as precise as possible with the available data. It was not possible to estimate actual exposure levels, because the air monitoring results could not be linked to specific jobs. Monitoring data were specific to shop, whereas the job titles could not be identified with shops. Consequently, to develop exposure estimates we had to rely on other sources of data, including worker compensation files, histories and telephone books of the facility, organisation charts, technical orders, and position descriptions. Several attempts were made using these sources to develop semiquantitative (low, medium, high) exposure estimates for all jobs for all chemicals, but none was successful because of the inability to link job to shop. It was also difficult to identify specific solvents used in particular jobs. Many solvents were purchased and used at any one time for various purposes. Also, shops changed solvents routinely and records of such changes were not kept. Because of this lack of specificity for most of the solvents and the inability to locate jobs in particular shops, an effort to estimate levels of exposure for most chemcials was not considered appropriate.

Trichloroethylene, however, was the chemical of interest at the outset of the study and for this reason a more detailed evaluation was necessary. Because low level exposures to mixed solvents so often occurred concomitantly with low level exposures to TCE, both types of exposures were the focus of the detailed evaluation of exposures. As a result, continuous and intermittent uses of mixed solvents and TCE, and frequent and infrequent peaks for TCE were identified.

As mentioned above, mixed solvents included a number of different solvents and inherently this means that the subjects identified as having exposure to mixed solvents differed as to which particular solvents they were exposed. Considering this category as a single substance in a mortality analysis, therefore, is somewhat misleading because it is unlikely that the solvents in this category all have the same effect on health. As a result of this mixture of relevant and irrelevant exposures, any risks of disease due to a single agent would be muted. This problem, however, is not different from that of one of the traditional epidemiological methods of analysisthat is, using employment in a plant as the determinant of exposure, because that approach is also likely to include people with different chemical exposures. Similarly, misclassification also occurs when estimating levels of exposure to mixed solvents as a single category but this problem is similar to the misclassification accepted when duration of employment is used to investigate exposure-response trends. The effect of both approaches (using mixed solvents and using duration of employment as a surrogate for a single exposure) is to depress an exposure-response association.

When quantitative estimates have not been possible in other studies, investigators ${ }^{1819}$ have 
developed semiquantitative categories (low, medium, and high) and assigned arbitrary weights ( 1 , 2 , and 3) to these categories. In this study, it was possible to estimate indices of exposure to TCE (table 4), which were assigned to reflect relative differences in exposure levels to TCE over time (indices of $0,200,400$, and 600). Although these weights were based on the monitoring data and the frequency exposures were likely to have occurred, and are considered to be an improvement over arbitrary scales such as 1,2 , and 3, they should not be interpreted as designating actual exposure levels.

This assessment of TCE provides the opportunity to evaluate potential effects of continuous and peak exposures. Either or both could be important in cancer mortality; for example, if cumulative exposures are critical, adverse health effects could result from relatively continuous exposure at low levels. Conversely, if adverse health effects occur only after exposure to a particular level, workers who have had peak exposures may be at increased risk.

As in most occupational studies, ${ }^{125-710-121819}$ exposures were assessed on the basis of job title, not person. This approach assumes that persons in a given job have equivalent exposure levels. In reality, workers holding the same job may have different exposure levels because of differences in individual work practices and microenvironments. ${ }^{20}$ None the less, the practise of using job title as the basis of exposure in epidemiological studies is adopted for two practical reasons. Monitoring data often do not identify the person being sampled and, when such information is available, the data are not numerous enough to allow the development of exposure estimates for each worker. The monitoring data in this study reflected these limitations. Also, estimating exposure levels for every person in each job would require information on work practices for each person in the study. Such information is rarely, if at all, available in industrial hygiene records and therefore could only be obtained from interviews with each subject. In this study, for example, it would have meant estimating exposures for 150000 jobs, over three times the number for which estimates were made. In any case, although use of job titles as the basis for assessing exposures may result in some misclassification, such an approach is likely to be more accurate than that based solely on plant or department.

In spite of severe limitations of the available data, information was uncovered in the position descriptions and other historical documents collected that allowed an evaluation of tasks associated with various jobs; this could then be used to assess exposures. The study emphasises the need for investigators performing exposure assessments to approach each situation with an open mind. When the more traditionally used records (for example, monitoring data or typical personnel records) are non-existent or are not in a usable form, other records may exist that, when used creatively, can be used to perform relatively precise exposure assessments. The study also stresses the need to evaluate job histories before the start of the study to ensure that exposures can be specifically identified from the jobs, or departments, or both.

The conclusions, recommendations, or other views expressed herein are those of the authors and do not necessarily represent the official views of the American Federation of Government Employees or the US Air Force, Department of Defence.

Requests for reprints to: Patricia A Stewart, CIH, National Cancer Institute, Executive Plaza North, Room 418 Rockville, MD 20892, USA.

1 Mancuso TF, Ciocco A, El-Attar AA. An epidemiological approach to the rubber industry: a study based on departmental experience. J Occup Med 1968;12:213-32.

2 Lloyd JW, Lundin FE, Redmond CK, Geiser PB. Long-term mortality study of steelworkers. IV. Mortality by work area. J Occup Med 1970;10:151-7.

3 Gamble J, Spirtas R. Job classification and utilisation of complete work histories in occupational epidemiology. J Occup Med 1976;18:399-404.

4 Corn M, Esmen NA. Workplace exposure zones for classification of employee exposures to physical and chemical agents. $A m$ Ind Hyg Assoc J 1979;50:47-57.

5 Stewart PA, Blair A, Cubit DA, et al. Estimating historical exposures to formaldehyde in a retrospective mortality study. Applied Industrial Hygiene 1986;1:34-41.

6 Lee AM, Fraumeni JF, Jr. Arsenic and respiratory cancer in man: An occupational study. JNCI 1968;42:1045-52.

7 Gibbs GW, Lachance $M$. Dust exposure in the chrysotile asbestos mines and mills of Quebec. Arch Environ Health 1972;24:189-97.

8 Esmen N. Retrospective industrial hygiene surveys. Am Ind Hyg Assoc J 1979;40:58-65.

9 Dement JM, Harris RL, Symons MJ, Shy CM. Exposures and mortality among chrysotile asbestos workers. Part I: Exposure estimates. Am J Ind Med 1983;4:399-419.

10 Greenburg RA, Tamburro $\mathrm{CH}$. Exposure indices for epidemiological surveillance of carcinogenic agents in an industrial chemical environment. J Occup Med 1981;23: 353-8.

11 Rice C, Harris RL, Lumsden JC, Symons MJ. Reconstruction of silica exposure in the North Carolina dusty trades. Am Ind Hyg Assoc J 1984;45:689-96.

12 Amandus HE, Wheeler R, Jankovic J, Tucker J. The morbidity and mortality of vermiculite miners and millers exposed to tremolite-actinolite: Part I. Exposure estimates. Am J Ind Med 1987;11:1-14.

13 Spirtas R, Stewart PA, Lee JS, et al. Retrospective cohort mortality study of workers at an aircraft maintenance facility. I Epidemiological results. Br J Ind Med 1991;48:515-30.

14 von Fischer W, ed. Paint and varnish technology. New York: Rheinhold Publishing Corporation, 1948:197-201, 237-63.

15 Elkins $H$. The chemistry of industrial toxicology, 2nd ed. New York: John Wiley and Sons Inc, 1959:191-3.

16. Hill US Air Force. Report of Industrial Hygiene Engineering Survey, vapor degreasers, maintenance directorate. Ogden, Utah: unpublished, March 1965.

17 Vigil TS. Bioenvironmental engineering survey of maintenance shops at Hill $A F B$. Hill US Air Force Base, Ogden, Utah: unpublished, March-June 1971.

18 Vaughan TL, Strader C, Davis S, Daling JR. Formaldehyde and cancers of the pharynx, sinus and nasal cavity: I. Occupational exposures. Int J Cancer 1986;38:677-84.

19 Gerin M, Siemiatycki J, Kemper H, Begin D. Obtaining occupational exposure histories in epidemiologic case-control studies. J Occup Med 1985;27:420-6.

20 Smith TJ, Hammond SK, Hallock M, Woskie SR. Exposure assessment for epidemiology: characteristics of exposure. Applied Industrial Hygiene (in press).

Accepted 7 January 1991 